\title{
Low Digit Ratio Predicts Early Age at Menarche in Colombian Schoolgirls
}

\author{
Anna S. Oberg, ${ }^{a}$ Eduardo Villamor ${ }^{a, b}$ \\ ${ }^{a}$ Department of Epidemiology, Harvard School of Public Health, Boston, MA, and ${ }^{b}$ Department of Epidemiology, School of Public Health, \\ University of Michigan, Ann Arbor, MI, USA
}

\begin{abstract}
Background: The ratio between the lengths of the second and fourth fingers (digit ratio: 2D :4D), a purported negative correlate of prenatal androgen exposure, has been inversely related to age at menarche. However, a recent study found high digit ratios in carriers of a single variant in the LIN28B gene, which has been linked to delayed menarche.

Methods: We investigated the association of digit ratio and age at menarche in 299 pre-menarcheal girls aged 5-12 years who participated in a longitudinal cohort study in Bogotá, Colombia. Finger lengths were measured at baseline and the occurrence of menarche was periodically ascertained over a median 32 months of follow-up. We used time-to-event analysis to estimate median ages at menarche as well as hazard ratios for menarche according to tertiles of the digit ratio for each hand.

Results: Estimated median age at menarche was lower for girls in the lowest digit ratio tertile of the right hand compared with those in the highest (12.0 vs. 12.3 years; $P$-value $=0.04)$. After adjustment for baseline age, heightand body mass index-for-age $z$-scores, the hazard of menarche was $86 \%$ higher in girls of the lowest digit ratio tertile (hazard ratio 1.9 [95\% confidence interval 1.2, 2.9]) compared with those in the highest digit ratio tertile of the right hand. No significant associations were found with the left hand.

Conclusions: Digit ratio was positively associated with age at menarche in this longitudinal investigation, consistent with results from a recent gene-linkage study.
\end{abstract}

Keywords: Anthropometry, Digit ratio, Menarche, Cohort studies.

Sexual dimorphism in the ratio between the index and ring finger lengths (2D : 4D; usually higher in males than females) manifests already in utero. ${ }^{1}$ It has been proposed that lower digit ratios indicate greater androgen exposure in utero, ${ }^{2}$ and studies have linked the digit ratio with a variety of reproductive, behavioural and health outcomes including fertility, ${ }^{2}$ sexual orientation, ${ }^{3,4}$ physical performance, ${ }^{5,6}$ spatial ability ${ }^{7}$ and autism. ${ }^{8}$ The direction and magnitude of these associations, however, has not been consistent across studies.

Because the hormonal intrauterine environment might influence the timing of pubertal development, ${ }^{9}$ recent investigations have addressed the association between the digit ratio and age at menarche. In students from the US ${ }^{10}$ and among British women, ${ }^{11}$ low

Correspondence: Anna Sara Oberg, Department of Epidemiology, Harvard School of Public Health, 677 Huntington Avenue, Boston, MA 02115, USA.

E-mail: oberg@hsph.harvard.edu digit ratio in the right hand was related to delayed age at menarche. In contrast, no association was reported between digit ratio in either hand and age at menarche in a retrospective study of post-menopausal Finnish women. ${ }^{12}$ In a recent study of Australian and British girls, the digit ratio was strongly associated with a single variant in the LIN28B gene; each copy of the minor allele was associated with a 0.006 increase in mean $2 \mathrm{D}: 4 \mathrm{D} .{ }^{13}$ As this variant has been linked to height ${ }^{14}$ and delayed age at menarche,,$^{15,16}$ the finding would suggest a positive association between digit ratio and age at menarche. ${ }^{13}$

Previous studies examining the relation of digit ratio and age at menarche have been cross-sectional or retrospective in nature; that is, the age at menarche has been self-reported at the time when the digit ratio was ascertained. In these studies it is not possible to determine the temporal relation between the exposure (digit ratio) and the outcome (age at menarche). We conducted a prospective study of pre-menarcheal 
schoolgirls from Bogotá, Colombia to investigate the potential association between the digit ratio and menarche.

\section{Methods}

\section{Study population}

The Bogotá School Children Cohort is an ongoing longitudinal study to investigate the nutrition and health status of school-age children. Details of the study design have been reported previously. ${ }^{17}$ In brief, 3202 children aged 5-12 years who were enrolled in the public primary school system of Bogotá, Colombia, were recruited into the study in February 2006. The public school system enrols a majority of children from low- and middle-income families in the city and the use of a cluster sampling technique ensured that the cohort was representative of this population. Information on sociodemographic characteristics and health habits of the children and their families was collected through a self-administered questionnaire that was sent to the parents at enrolment (response rate $82 \%$ ). In the following weeks, research assistants made school visits to obtain anthropometric measurements in all participating children, including height and weight, according to standardised procedures. ${ }^{18}$ In addition, we measured finger lengths in a random sample of 652 children with the use of digital calipers (Digimatic Caliper CD-6" CX, 500-171 series, Mitutoyo Corporation, Aurora, IL, USA) with 0.01-mm precision. Each finger of both hands was measured twice by the same observer. The fixed jaw of the caliper was placed midline of the basal crease of each finger and the sliding jaw was extended to the fingertip without exerting pressure. Additional follow-up visits occurred in June and November 2006, and once annually thereafter. If children were absent from school on the day of the assessment, they were visited at home. At each of these assessments, girls were asked whether or not they had started menstruating; among those who provided an affirmative response we recorded the date of the first menstrual period.

The parents or primary care providers provided written informed consent before enrolment. The study protocol was approved by the Ethics Committee of the National University of Colombia Medical School; the Institutional Review Board at the Harvard School of Public Health approved the use of data from the study.

\section{Statistical analyses}

There were 321 girls in the subsample with finger length measurements. Seven of them had already experienced menarche at the time of enrolment and 15 were lost to follow-up; thus the final sample size for analysis was 299. The primary exposure of interest was the digit ratio, estimated as the ratio of the index (second) and ring (fourth) fingers of each hand. The primary end-point was whether menarche occurred during follow-up and at what age.

We first examined the reliability of the finger ratio measurements by calculating intraclass correlations (ICCs) between the two assessments of each finger, separately for each hand (Table 1). Because reliability was high, we estimated digit ratios using the mean values from the two measurements of each finger.

Potential confounders were investigated by comparing the distribution of digit ratios according to

Table 1. Finger measurements $(\mathrm{mm})$ in 299 girls of the Bogotá School Children Cohort

\begin{tabular}{|c|c|c|c|c|c|}
\hline & \multicolumn{2}{|c|}{ Mean (SD) } & \multicolumn{2}{|c|}{ Variance } & \multirow[b]{2}{*}{ ICC } \\
\hline & Measure 1 & Measure 2 & Between & Within & \\
\hline \multicolumn{6}{|l|}{ Right hand } \\
\hline 2: index finger & $54.2(5.6)$ & $54.2(5.6)$ & & & \\
\hline 4: ring finger & $56.9(5.5)$ & $56.9(5.4)$ & & & \\
\hline $2: 4$ digit ratio & $0.952(0.034)$ & $0.953(0.033)$ & 0.0010 & 0.00013 & 0.88 \\
\hline \multicolumn{6}{|l|}{ Left hand } \\
\hline 2: index finger & $54.0(5.5)$ & $54.0(5.5)$ & & & \\
\hline 4: ring finger & $56.7(5.4)$ & $56.7(5.3)$ & & & \\
\hline $2: 4$ digit ratio & $0.953(0.037)$ & $0.953(0.035)$ & 0.0012 & 0.00015 & 0.88 \\
\hline
\end{tabular}

ICC, intraclass correlation coefficient. 
baseline characteristics of the girls and their mothers. These included the girl's age, handedness, anthropometric status and birthweight; the mother's age, height and body mass index (BMI); and the household's socio-economic status (SES). SES was determined according to the local government's classification of households that determines public service fees. We calculated height- and BMI-for-age standardised $z$-scores according to the World Health Organization reference. ${ }^{19}$ Height-for-age $z$-score was classified as $<-1,-1$ to $<0$ and $\geq 0$ as the prevalence of stunting ( $z$-score $<2$ ) in this population is $<10 \%{ }^{20}$ Weight status was categorised as thin, normal or overweight according to BMI-for-age, based on the recommendations of the International Obesity Task Force. ${ }^{21}$ We first compared the distributions of digit ratios by categories of these potential confounders with the use of ANOva F-tests.

Next, we conducted time-to-event analyses with the use of Kaplan-Meier curves. In these analyses, the time to event was age at menarche in decimal years, estimated from the date of menarche and the date of birth. Girls who did not have menarche during follow-up were censored at the last time they reported that menarche had not occurred. Median age at menarche was estimated from the Kaplan-Meier curves for each tertile of the digit ratio, and these were compared with the log-rank test. Next, we estimated adjusted hazard ratios (HR) with the use of Cox proportional hazards models. In these, the occurrence of menarche was modelled with age in decimal years as the underlying timescale, and tertiles of the digit ratio as predictors. Adjustment covariates included known correlates of menarche or baseline characteristics that were associated with the digit ratio at $P<0.10$ in univariate analyses. The girl's age at baseline and predictors that remained significantly associated with menarche $(P<0.05)$ after multiple adjustment were retained in the final models. Because the associations of weight and height with age at menarche are fairly linear, ${ }^{22}$ these covariates were also considered as continuous. The proportional hazards assumption was evaluated by introducing terms for interaction between age and the covariates in the model. All analyses were carried out for the digit ratio of each hand separately. Analyses were performed in SAS version 9.2 (SAS Institute Inc., Cary, North Carolina, USA). Kaplan-Meier plots were produced in R statistical software version 2.12.0 (The $\mathrm{R}$ project for statistical computing - www.r-project.org).

\section{Results}

Mean age of participating girls at baseline was $8.8 \pm 1.7$ years. ICCs were high for the digit ratios of both hands, indicating that measurement error was not substantial (Table 1).

We next examined the distributions of digit ratios according to baseline characteristics separately for each hand. Digit ratios were higher in older compared with younger girls, and also in thin and overweight girls compared with those with normal weight (Table 2). Left-handedness, weight at birth and heightfor-age $z$-score at the time of recruitment, or the family's SES were not associated with digit ratio. Maternal BMI was positively associated with the girl's left hand digit ratio (Table 2).

The median length of follow-up was 32 months (interquartile range: 20-44); it was not related to the digit ratio. During this time, 141 girls $(47 \%)$ experienced menarche. Estimated median [95\% confidence interval, $\mathrm{CI}]$ age at menarche was $12.2[12.0,12.3]$ years overall. The cumulative probability curve of menarche for girls in the lowest tertile of digit ratio in the right hand was consistently above those for the higher tertiles (Figure 1). Estimated median age at menarche was the lowest for girls in the lowest right hand digit ratio tertile (12.0 years) compared with those in the mid- (12.2 years) or highest tertiles (12.3 years) respectively (log-rank test $P$-value $=0.04$; Figure 1$)$. There was no significant association between digit ratio of the left hand and age at menarche; estimated median age at menarche in the lowest, mid- and highest tertiles were, respectively, 12.0, 12.3 and 12.2 years (log-rank test $P$-value $=0.17$; Figure 2 ).

We next modelled the occurrence of menarche according to digit ratio tertiles for each hand with the use of Cox regression models (Table 3). The hazard rate of menarche was the highest among girls in the lowest right hand digit ratio tertile ( $\mathrm{HR}=1.7$ [95\% CI 1.1, 2.5] compared with girls in the highest tertile). Adjustment for age and anthropometric indicators at baseline (height- and BMI-for-age $z$-score) increased the magnitude of the association slightly ( $H R=1.9$ [95\% CI 1.2, 2.9]), whereas adjustment for maternal BMI or family SES had no influence on the estimates (results not shown). The associations were not statistically significant for the left-hand digit ratio. Tests of potential violations to the proportional hazard assumption were negative for the digit ratio and all covariates considered. 
Table 2. Digit ratio and estimated age at menarche according to baseline characteristics in 299 girls from the Bogotá School Children Cohort

\begin{tabular}{|c|c|c|c|c|c|c|c|c|}
\hline \multirow[b]{3}{*}{ Characteristic } & \multirow[b]{3}{*}{$n^{\mathrm{a}}$} & \multicolumn{4}{|c|}{ Digit ratio } & \multicolumn{3}{|c|}{ Menarche } \\
\hline & & \multicolumn{2}{|c|}{ Right hand } & \multicolumn{2}{|c|}{ Left hand } & \multirow{2}{*}{$\begin{array}{l}\text { Menarche } \\
\text { during } \\
\text { follow-up }(n)\end{array}$} & \multirow{2}{*}{$\begin{array}{c}\text { Median } \\
\text { estimated age } \\
\text { at menarche }\end{array}$} & \multirow[b]{2}{*}{$P^{\mathrm{d}}$} \\
\hline & & Mean & $P^{\mathrm{b}}$ & Mean & $P^{\mathrm{b}}$ & & & \\
\hline Age, years & & & 0.005 & & 0.08 & & & 0.06 \\
\hline 5-6 & 61 & 0.944 & & 0.947 & & 3 & - & \\
\hline $7-8$ & 101 & 0.950 & & 0.950 & & 25 & 12.3 & \\
\hline $9-10$ & 113 & 0.954 & & 0.956 & & 91 & 12.1 & \\
\hline $11-12$ & 24 & 0.972 & & 0.966 & & 22 & 12.5 & \\
\hline Left-handedness & & & 0.93 & & 0.11 & & & 0.51 \\
\hline No & 213 & 0.953 & & 0.952 & & 111 & 12.2 & \\
\hline Yes & 26 & 0.952 & & 0.964 & & 15 & 12.0 & \\
\hline Height-for-age $z^{\mathrm{e}}$ & & & 0.14 & & 0.17 & & & $<0.0001$ \\
\hline$<-1$ & 110 & 0.950 & & 0.951 & & 43 & 12.5 & \\
\hline-1 to $<0$ & 108 & 0.950 & & 0.950 & & 55 & 12.1 & \\
\hline$\geq 0$ & 75 & 0.959 & & 0.960 & & 39 & 11.8 & \\
\hline Weight status ${ }^{\mathrm{f}}$ & & & 0.16 & & 0.04 & & & 0.10 \\
\hline Thin & 37 & 0.960 & & 0.962 & & 22 & 12.2 & \\
\hline Normal & 218 & 0.950 & & 0.950 & & 93 & 12.2 & \\
\hline Overweight & 38 & 0.956 & & 0.961 & & 22 & 12.0 & \\
\hline Birthweight $<2500 \mathrm{~g}$ & & & 0.40 & & 0.72 & & & 0.15 \\
\hline No & 210 & 0.952 & & 0.952 & & 96 & 12.2 & \\
\hline Yes & 22 & 0.946 & & 0.954 & & 13 & 12.0 & \\
\hline Mother's age, years & & & 0.03 & & 0.41 & & & 0.59 \\
\hline $20-29$ & 60 & 0.942 & & 0.946 & & 24 & 11.8 & \\
\hline $30-34$ & 81 & 0.954 & & 0.955 & & 38 & 12.2 & \\
\hline $35-39$ & 64 & 0.959 & & 0.954 & & 26 & 12.3 & \\
\hline$\geq 40$ & 74 & 0.953 & & 0.955 & & 42 & 12.1 & \\
\hline Mothers height, $\mathrm{cm}$ & & & 0.18 & & 0.76 & & & 0.36 \\
\hline$<154$ & 54 & 0.949 & & 0.953 & & 26 & 12.0 & \\
\hline 154 to $<158$ & 72 & 0.959 & & 0.953 & & 33 & 12.3 & \\
\hline 158 to $<162$ & 62 & 0.954 & & 0.956 & & 28 & 12.3 & \\
\hline$\geq 162$ & 77 & 0.948 & & 0.950 & & 34 & 12.2 & \\
\hline Mother's BMI, kg/m² & & & 0.28 & & 0.02 & & & 0.81 \\
\hline$<18.5$ & 9 & 0.939 & & 0.930 & & 5 & 12.0 & \\
\hline $18.5-24.9$ & 163 & 0.952 & & 0.951 & & 72 & 12.1 & \\
\hline $25-29.9$ & 64 & 0.956 & & 0.959 & & 27 & 12.2 & \\
\hline$\geq 30$ & 24 & 0.961 & & 0.965 & & 14 & 12.2 & \\
\hline Household SESg & & & 0.91 & & 0.07 & & & 0.30 \\
\hline $1+2$ (lowest) & 62 & 0.954 & & 0.955 & & 23 & 12.4 & \\
\hline 3 & 199 & 0.952 & & 0.955 & & 103 & 12.0 & \\
\hline 4 & 38 & 0.952 & & 0.941 & & 15 & 12.3 & \\
\hline
\end{tabular}

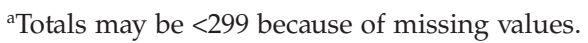

${ }^{\mathrm{b}}$ ANOVA F-test.

${ }^{\mathrm{C}}$ Estimated from Kaplan-Meier survival curves.

${ }^{\mathrm{d}}$ Log-rank test.

eAge-standardised according to the World Health Organization's reference. ${ }^{19}$

${ }^{\mathrm{f}}$ According to body mass index (BMI)-for-age criteria by the International Obesity Task Force. ${ }^{21}$

'Socio-economic status according to the local government's classification. 


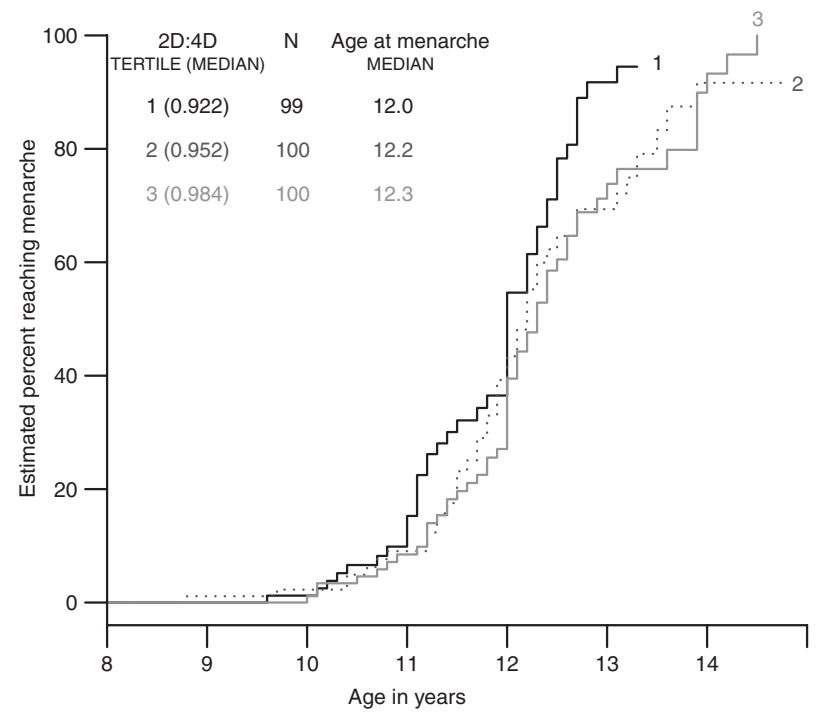

Figure 1. Menarche according to tertiles of 2D : 4D (right hand).

\section{Comments}

Digit ratio was positively associated with age at menarche in this prospective study of schoolgirls from Bogotá, Colombia. The finding is in contrast with previously reported associations of low digit ratio with delayed age at menarche. ${ }^{10,11}$ The inconsistency of the findings could partly be a result of methodological differences between the studies. In one of the studies finger length measurements were obtained by the participants themselves, according to instructions

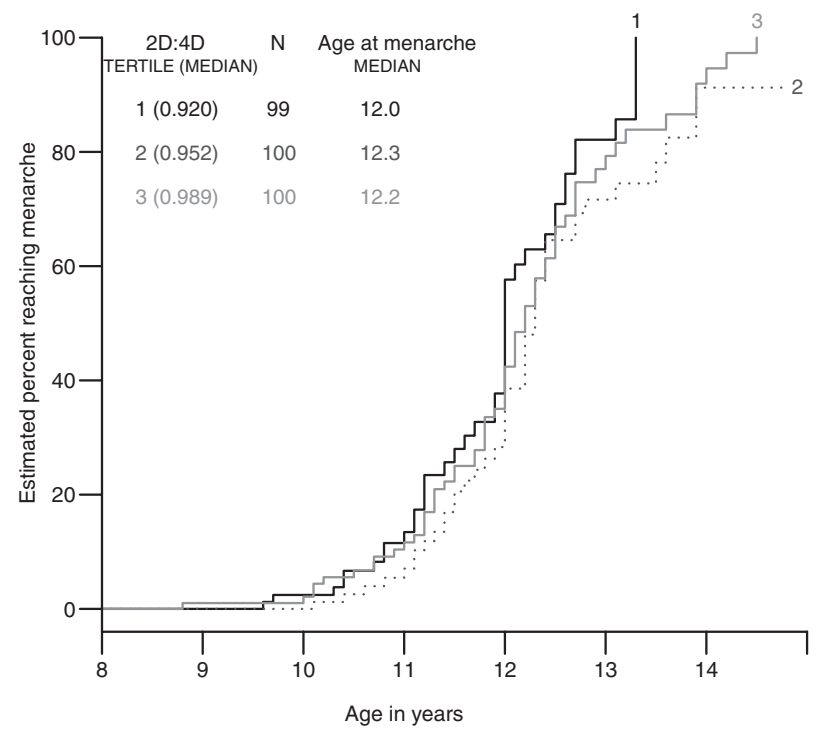

Figure 2. Menarche according to tertiles of 2D : 4D (left hand).

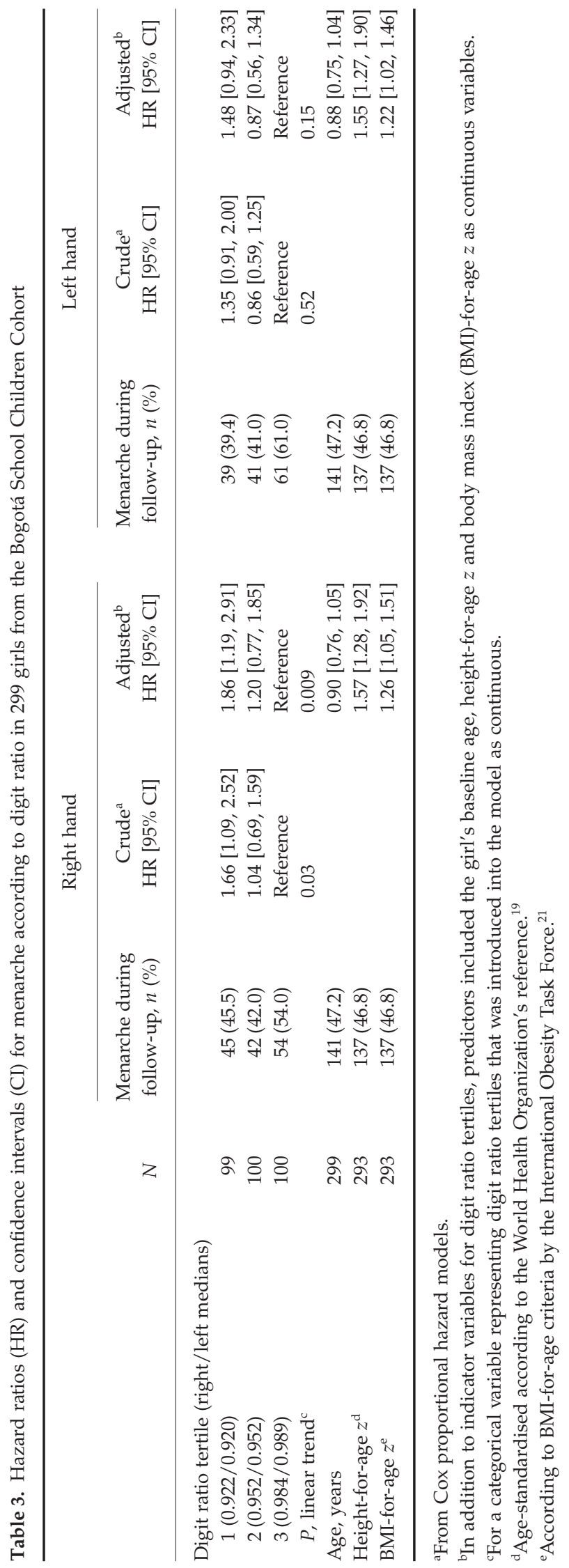

(C) 2012 Blackwell Publishing Ltd 
provided in a questionnaire. ${ }^{11}$ Because determinations of finger length are subject to substantial measurement error, the validity of self-measurements is questionable. In contrast, our study used finger length measurements made in duplicate by trained research personnel, and high ICCs between measurements indicated that intra-rater reliability was adequate. None of the previous investigations had a longitudinal design; digit ratios were measured after menarche had occurred, and age at menarche was ascertained by recall. ${ }^{10,11}$ We used a longitudinal design in which finger ratios were measured before the occurrence of menarche, largely precluding potential reverse causation bias. Ascertaining the occurrence of menarche in a prospective manner should also serve to minimise recall bias.

Albeit opposite to the results of initial studies, our findings are consistent with those of a recent report showing that minor allele carriers of a single variant in a gene (LIN28B) related to delayed menarche ${ }^{15}$ had high digit ratios. ${ }^{13}$ Also, the direction of the association in our study is consistent with that reported in an investigation of 282 Finnish women, in whom a standard deviation increase of the digit ratio was related to a non-statistically significant $14 \%$ lower hazard of menarche. ${ }^{12}$

The digit ratio was first proposed as a proxy for prenatal androgen exposure from the observation that sexual dimorphism in this trait appears early in life. ${ }^{2}$ Genetic conditions that increase or decrease early exposure to androgens have also been related to lower or higher digit ratios respectively. ${ }^{23,24}$ Thus, previous findings of an inverse association between digit ratio and age at menarche were interpreted as suggesting that prenatal androgen exposure may delay sexual maturation in females. ${ }^{10}$ Some evidence from animal experiments lent support to this view, as exposure of rhesus monkeys or sheep to prenatal testosterone have induced delayed age at menarche ${ }^{25}$ and ovulatory fail$u^{2} e^{26}$ respectively. Nevertheless, information from epidemiological studies to support a direct link between prenatal exposure to sex hormones and the timing of puberty is limited. Also sparse is the evidence of a direct effect of the prenatal hormonal environment on the digit ratio. In a study of 30 mother-infant pairs, there were no correlations between testosterone or oestrogen levels in amniotic fluid and the children's digit ratio at 2 years of age; however, an inverse correlation was noted with the testosterone/oestrogen ratio. $^{27}$
Although digit ratios have been related to postnatal conditions that purportedly represent the effect of early differential exposures to sex hormones including sperm count, ${ }^{2}$ sexual orientation, ${ }^{3,4}$ physical performance, ${ }^{5,6}$ spatial ability ${ }^{7}$ and aggressive behaviour, ${ }^{28}$ the associations reported do not consistently follow the direction expected if a low digit ratio were to represent high exposure to androgens in early life. For example, homosexuality has been linked to both high and low digit ratio in different populations. ${ }^{4}$ Also, a recent meta-analysis of studies of the digit ratio and spatial ability found that their correlation was negligible. ${ }^{7}$

While androgens play an indisputable role in the regulation of sexual differentiation and maturation, their action likely depends on the timing of exposure as well as their interactions with other sex hormones. Using the digit ratio as a proxy for prenatal hormone exposure may be limited by its inability to differentiate the potential effect of exposure in utero from that occurring postnatally. ${ }^{29}$ There are reports indicating a correlation between the digit ratio and testosterone or oestradiol serum levels in adults, ${ }^{2,30}$ but these have also been contradicted by a meta-analysis showing no such associations. ${ }^{29}$ Lastly, the digit ratio does not appear to be constant throughout life. In line with findings from previous longitudinal assessments, ${ }^{31,32}$ we noted a positive association of the digit ratio with baseline age in cross-sectional analyses. Whether agerelated differences are a result of postnatal hormonal effects has not been established.

We observed stronger associations in the right than in the left hand. This finding is consistent with many of the previous reports that examined the digit ratio in relation to traits and health outcomes. ${ }^{2,5,10,11,30}$ While potential mechanisms are poorly understood, the male form of sexually dimorphic traits appears to be most strongly expressed on the right side of the body. ${ }^{33,34}$ It has also been proposed that the right hand may be more sensitive to the effect of testosterone in utero. $^{35}$

There are some limitations to our study. Follow-up was not sufficiently long as to allow us to observe the occurrence of menarche in all participants; this could have decreased statistical power. Also, we relied on the ascertainment of the exposure at a single time point (at start of follow-up), and serial measurements in children suggest that the digit ratio increases with age. ${ }^{31,32}$ However, other studies indicate that sex differences in the digit ratio remain stable over time, and 
Spearman correlation coefficients for repeated measures of digit ratios are high. ${ }^{32}$ It is thus possible that these changes occur systematically, without altering the ranking of girls according to their digit ratio over time. Finally, although ICCs were high, there was no formal evaluation of inter-observer variation in the measurement of finger lengths.

In summary, a low digit ratio was associated with earlier menarche in this longitudinal study. Whether the digit ratio is a valid proxy of prenatal hormone exposure in the prediction of postnatal health outcomes requires further evaluation in longitudinal investigations.

\section{Acknowledgements}

The Bogotá School Children Cohort is currently sponsored by the ASISA Research Fund at the University of Michigan and the Center for Global Health at the University of Michigan.

\section{References}

1 Malas MA, Dogan S, Evcil EH, Desdicioglu K. Fetal development of the hand, digits and digit ratio (2D : 4D). Early Human Development 2006; 82:469-475.

2 Manning JT, Scutt D, Wilson J, Lewis-Jones DI. The ratio of 2nd to 4th digit length: a predictor of sperm numbers and concentrations of testosterone, luteinizing hormone and oestrogen. Human Reproduction 1998; 13:3000-3004.

3 Rahman Q, Wilson GD. Sexual orientation and the 2nd to 4th finger length ratio: evidence for organising effects of sex hormones or developmental instability?

Psychoneuroendocrinology 2003; 28:288-303.

4 Manning JT, Robinson SJ. 2nd to 4th digit ratio and a universal mean for prenatal testosterone in homosexual men. Medical Hypotheses 2003; 61:303-306.

5 Manning JT, Morris L, Caswell N. Endurance running and digit ratio (2D : 4D): implications for fetal testosterone effects on running speed and vascular health. American Journal of Human Biology 2007; 19:416-421.

6 Manning JT, Hill MR. Digit ratio (2D : 4D) and sprinting speed in boys. American Journal of. Human Biology 2008; 21:210-213.

7 Puts DA, McDaniel MA, Jordan CL, Breedlove SM. Spatial ability and prenatal androgens: meta-analyses of congenital adrenal hyperplasia and digit ratio (2D : 4D) studies. Archives of Sexual Behavior 2008; 37:100-111.

8 Manning JT, Baron-Cohen S, Wheelwright S, Sanders G. The 2nd to 4th digit ratio and autism. Developmental Medicine and Child Neurology 2001; 43:160-164.

9 Epplein M, Novotny R, Daida Y, Vijayadeva V, Onaka AT, Le Marchand L. Association of maternal and intrauterine characteristics with age at menarche in a multiethnic population in Hawaii. Cancer Causes and Control 2010; 21:259-268.

10 Matchock RL. Low digit ratio (2D : 4D) is associated with delayed menarche. American Journal of Human Biology 2008; 20:487-489.

11 Manning JT, Fink B. Is low digit ratio linked with late menarche? Evidence from the BBC internet study. American Journal of Human Biology 2011; 23:527-533.

12 Helle S. Does second-to-fourth digit length ratio (2D : 4D) predict age at menarche in women? American Journal of Human Biology 2010; 22:418-420.

13 Medland SE, Zayats T, Glaser B, Nyholt DR, Gordon SD, Wright MJ, et al. A variant in LIN28B is associated with 2D : 4D finger-length ratio, a putative retrospective biomarker of prenatal testosterone exposure. American Journal of Human Genetics 2010; 86:519-525.

14 Lettre G, Jackson AU, Gieger C, Schumacher FR, Berndt SI, Sanna $S$, et al. Identification of ten loci associated with height highlights new biological pathways in human growth. Nature Genetics 2008; 40:584-591.

15 He C, Kraft P, Chen C, Buring JE, Pare G, Hankinson SE, et al. Genome-wide association studies identify loci associated with age at menarche and age at natural menopause. Nature Genetics 2009; 41:724-728.

16 Perry JR, Stolk L, Franceschini N, Lunetta KL, Zhai G, McArdle PF, et al. Meta-analysis of genome-wide association data identifies two loci influencing age at menarche. Nature Genetics 2009; 41:648-650.

17 Arsenault JE, Mora-Plazas M, Forero Y, Lopez-Arana S, Baylin A, Villamor E. Hemoglobin concentration is inversely associated with erythrocyte folate concentrations in Colombian school-age children, especially among children with low vitamin B12 status. European Journal of Clinical Nutrition 2009; 63:842-849.

18 Lohman TG, Roche AF, Martorell R. Anthropometric Standardization Reference Manual. Champaign, IL: Human Kinetics Books, 1988.

19 de Onis M, Onyango AW, Borghi E, Siyam A, Nishida C, Siekmann J. Development of a WHO growth reference for school-aged children and adolescents. Bulletin of the World Health Organization 2007; 85:660-667.

20 McDonald CM, Baylin A, Arsenault JE, Mora-Plazas M, Villamor E. Overweight is more prevalent than stunting and is associated with socioeconomic status, maternal obesity, and a snacking dietary pattern in school children from Bogotá, Colombia. Journal of Nutrition 2009; 139:370-376.

21 Cole TJ, Flegal KM, Nicholls D, Jackson AA. Body mass index cut offs to define thinness in children and adolescents: international survey. BMJ (Clinical Research Ed.) 2007; 335:194-197.

22 Villamor E, Marin C, Mora-Plazas M, Baylin A. Vitamin D deficiency and age at menarche: a prospective study. American Journal of Clinical Nutrition 2011; 94:1020-1025.

23 Berenbaum SA, Bryk KK, Nowak N, Quigley CA, Moffat S. Fingers as a marker of prenatal androgen exposure. Endocrinology 2009; 150:5119-5124. 
24 Brown WM, Hines M, Fane BA, Breedlove SM. Masculinized finger length patterns in human males and females with congenital adrenal hyperplasia. Hormones and Behavior 2002; 42:380-386.

25 Goy RW, Bercovitch FB, McBrair MC. Behavioral masculinization is independent of genital masculinization in prenatally androgenized female rhesus macaques. Hormones and Behavior 1988; 22:552-571.

26 Manikkam M, Steckler TL, Welch KB, Inskeep EK, Padmanabhan V. Fetal programming: prenatal testosterone treatment leads to follicular persistence/luteal defects; partial restoration of ovarian function by cyclic progesterone treatment. Endocrinology 2006; 147:1997-2007.

27 Lutchmaya S, Baron-Cohen S, Raggatt P, Knickmeyer R, Manning JT. 2nd to 4th digit ratios, fetal testosterone and estradiol. Early Human Development 2004; 77:23-28.

28 Hampson E, Ellis CL, Tenk CM. On the relation between 2D : 4D and sex-dimorphic personality traits. Archives of Sexual Behavior 2008; 37:133-144.

29 Honekopp J, Bartholdt L, Beier L, Liebert A. Second to fourth digit length ratio (2D : 4D) and adult sex hormone levels: new data and a meta-analytic review.

Psychoneuroendocrinology 2007; 32:313-321.
30 McIntyre MH, Chapman JF, Lipson SF, Ellison PT. Index-to-ring finger length ratio $(2 \mathrm{D}: 4 \mathrm{D})$ predicts levels of salivary estradiol, but not progesterone, over the menstrual cycle. American Journal of Human Biology 2007; 19:434-436.

31 McIntyre MH, Ellison PT, Lieberman DE, Demerath E, Towne B. The development of sex differences in digital formula from infancy in the Fels Longitudinal Study. Proceedings of the Royal Society of London. Series B. Biological Sciences 2005; 272:1473-1479.

32 Trivers R, Manning J, Jacobson A. A longitudinal study of digit ratio (2D : 4D) and other finger ratios in Jamaican children. Hormones and Behavior 2006; 49:150-156.

33 Tanner JM. Fetus into Man: Physical Growth from Conception to Maturity. Cambridge, MA: Harvard University Press, 1990.

34 Mittwoch U. Genetics of mammalian sex determination: some unloved exceptions. Journal of Experimental Zoology 2001; 290:484-489.

35 Honekopp J, Watson S. Meta-analysis of digit ratio 2D : 4D shows greater sex difference in the right hand. American Journal of Human Biology 2010; 22:619-630. 